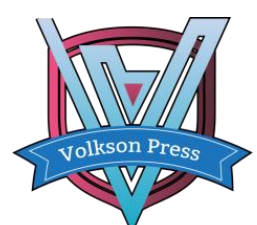

Contents List available at VOLKSON PRESS Intelligent Computing and Information Engineering (ICIE )

DOI : http://doi.org/10.26480/icie.01.2017.04.07

Journal Homepage: : https://www.intelcomp-design.com/

\title{
Application of Computer Technology in Hemodialysis Management System
}

Xiaowang CHEN, Yucheng SU*, Mingwei HUANG

Affiliated Hospital of Guilin Medical University, China

*email:24547350@qq.com

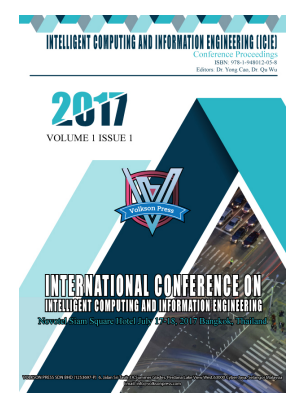

This is an open access article distributed under the Creative Commons Attribution License, which permits unrestricted use, distribution, and reproduction in any medium, provided the original work is properly cited

\section{ARTICLE DETAILS}

\section{Article History:}

Received 12 May2017

Accepted 12 July 2017

Available online 14 September 2017

Keywords:

Hemodialysis, management system, computer, data analysis.

\section{ABSTRACT}

In recent years, the number of patients with kidney disease in China has increased rapidly, and the number and size of hemodialysis centers have increased. At present, the hemodialysis work is facing more patients, equipment, medical staff, manual records, the machine on the complex plight of qualifying. Strengthen the hemodialysis center safety and quality management, and promote the development of hemodialysis technology, has become a hospital manager, doctors, nurses great challenge. This paper proposes an easy-to-use hemodialysis management system, which mainly follows the clinical business process of hospital hemodialysis center, manages and monitors hemodialysis treatment from patient management, dialysis schedule preparation, patient treatment scheduling, To facilitate the medical staff to grasp the most timely and accurate information, while eliminating the need for a large number of cumbersome written records, and solve the storage of various types of information. The main features of the system include the combination of clinical business, complete from the patient regis tration to the final quality control of the whole process of digital management processes, reduce the labor intensity of medical staff, improve the quality of medical care.

\section{Introduction}

Because hemodialysis patients are different from those in outpatient and ward patients, they require long-term repeated treatment, so the number of documents to be recorded in the hemodialysis center is large and large, but the data record of the hemodialysis center has been in the form of manual recording, not only low efficiency, and easy to go wrong. With the development of computer technology, the hospital information management system (HIS) has entered into all aspects of the hospital, the information system for medical equipment management software is also increa sing, but for hemodialysis management information platform is relatively small [1]. This paper mainly discusses the information system of hemodialysis center work. The system is based on the actual work of hematology center in our hospital. It is realized after fully understanding the needs of medical care and engineering and technical personnel [2]. The system for the nature of the work of various types of staff divided into different functional mo dules, through the system doctors can achieve the creation and management of patient information, you can handle the doctor's advice and the development of dialysis program; nurses can call patient information and doctor's advice, the implementation of medical advice and Monitoring and control of the patient during the dialysis treatment of patient signs data and dialysis machine status data; engineering and technical personnel can effectively control the equipment of the hemodialysis center; the system also uses serial communication way to hemodialysis equipment, a variety of parameters focused on the software The central station and stored records to the nursing module; department management staff can be hemodialysis center supplies and department staff to manage [3].

The main function of the system is to achieve the hemodialysis center work information, the system to our hospital hemodialysis center actual work process as a blueprint for the design. The system platform can facilitate the management and query of patient information, doctors, nurses, engineering and technical personnel and departmental managers were logged on the system, have their own rights to their respective work [4]. Doctors can easily under the doctor's advice, nurses can record the treatment data, engineering and technical personnel can manage the equipment, department managers can manage department affairs. Specifically, the information platform can be achieved:

(1) With a graphical man-machine interface, easy to operate.

(2) According to the professional characteristics of each department hierarchical management, each user has a different use of authority, different levels of user operations are different but there are interrelated. As mentioned earlier, doctors, nurses, engineering and technical personnel, department management personnel into their respective operating modules, each according to their actual work to operate, but between the various modules through the basic information of patients with each other.

(3) To achieve serial communication, hemodialysis equipment can be concentrated on the treatment parameters displayed on the monitoring platform, and can be recorded and stored.

(4) Can be on the department of equipment maintenance and management, hemodialysis supplies and departments to conduct a simple management, to achieve the entire hemodialysis center work information.

\section{SYSTEM DESIGN}

\subsection{Demand analysis}

Hemodialysis information platform should be easy to operate, stable and reliable, scalable, easy maintenance and other aspects of performance.

\subsubsection{Easy to operate}


System requirements with simple operation, easy to use features, the system requires a very friendly human-computer interaction interface, and in line with people's operating habits, system users can quickly skilled use of the information platform.

\subsubsection{Stable and reliable}

The system is mainly in the processing and recording of patients, equipment, supplies and other data information, so the system must ensure that the process of running a stable and reliable, as much as possible in the event of timely error, and can automatically backup data.

\subsubsection{Extensible, easy to maintain}

In order to meet the requirements of system change, the software design should be used in the design of the scheme. At the same time, the system can be extended and easy to be maintained so that after the system is completed, the user can use the simple method and the low cost to realize the system expansion with maintenance.

\section{SYSTEM STRUCTURE}

The hemodialysis information system network architecture mainly divides the server and the client two parts. The server has two types of data server and communication server. The database server stores the patient's hemodialysis medical records and related data. The communication server is responsible for the real-time data collection of the hemodialysis equipment. It is used as a blood pipe for the hemodialysis equipment and information system. The collected data is transmitted to the hemodialysis information system. As shown in Figure 1.

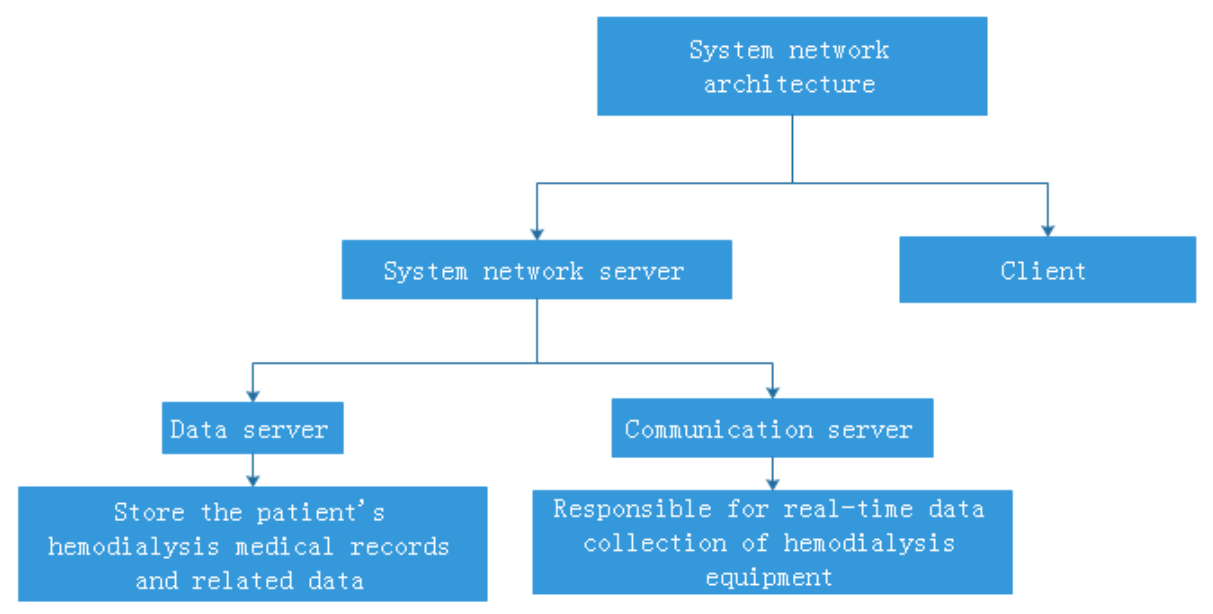

Figure 1: Hemodialysis information system network architecture

Hemodialysis information system software logic architecture is divided into security authentication layer, business function layer, system service layer, data layer.

\subsection{Safety certification layer}

The security certification layer handles the security certification of the operator and whether the client is secure, ensuring the legitimate access of the authorized user, blocking the entry of the illegal user and the terminal, and also protecting the privacy of the patient.

\subsection{Business function layer}

Business function layer to complete the system's business functions, full support for hemodialysis workflow to meet the operator's schedule business processing needs, but also according to the needs of the operator to adjust.

\subsection{System service layer}

The system service layer serves as the background service function running on the server side, and provides the back-office service support for the service layer, such as the data access and the receiving of the instrument and equipment data.

\subsection{Data layer}

The data layer is responsible for the storage of business data. The storage of data is not limited to the database system, but also the file data storage mode.

\section{SYSTEM FUNCTIONS}

The hemodialysis information system functions are:

(1) Patient management

Completion of patient-related information retrieval and maintenance, including patient list, file management, medical records, patient scheduling, patient appointment and so on.

(2) Dialysis management

The hemodialysis process is mainly based on the management of the relevant information during the dialysis process, including dialysis records, dialysis monitoring, auxiliary inspection and test results, doctor management, medical records management, dialysis announcement, which is the key function of the system.

(3) Transaction management 
Completion of human, financial, and other ancillary business management. Including staff scheduling, equipment maintenance, cost statistics, workload inquiries.

\subsection{Data interface}

Hemodialysis information system and the hospital's other information systems have a lot of contact, the formation of a large number of system interfaces:

(1) Interface with the HIS.

For example, the new patient registration first from the HIS database to read the basic information of patients, in addition to the basic information of patients, there are costs and medication information and the basic information of doctors and nurses, as the basic data to run the system, through the interface Direct access to coexist hemodialysis information system, to achieve data sharing;

(2) Interface with the LIS.

Hemodialysis and blood tests are inseparable, the system and the LIS interface, the test results can be based on the patient's hospital number or outpatient number directly transferred to the hemodialysis information system, need to write hemodialysis medical records can also be directly inserted into the system, Personnel to record the workload

(3) Interface with the hospital EMR.

If it is hospitalized patients, through the interface with the hospital EMR, can be directly inquired into the patient's hospital EMR, of course, is limited to medical records browsing authority;

(4) Hemodialysis equipment interface.

This is the system's most important interface, through the device interface, directly to the equipment related information to read the system, and conversion, directly into the hemodialysis system.

\subsection{System Design}

According to the demand analysis and the division of the responsibilities of medical staff in our hospital, the system should also have the doctor function module, the nurse function module, the engineering technician function module and the administrator function module, as shown in Figure 2.

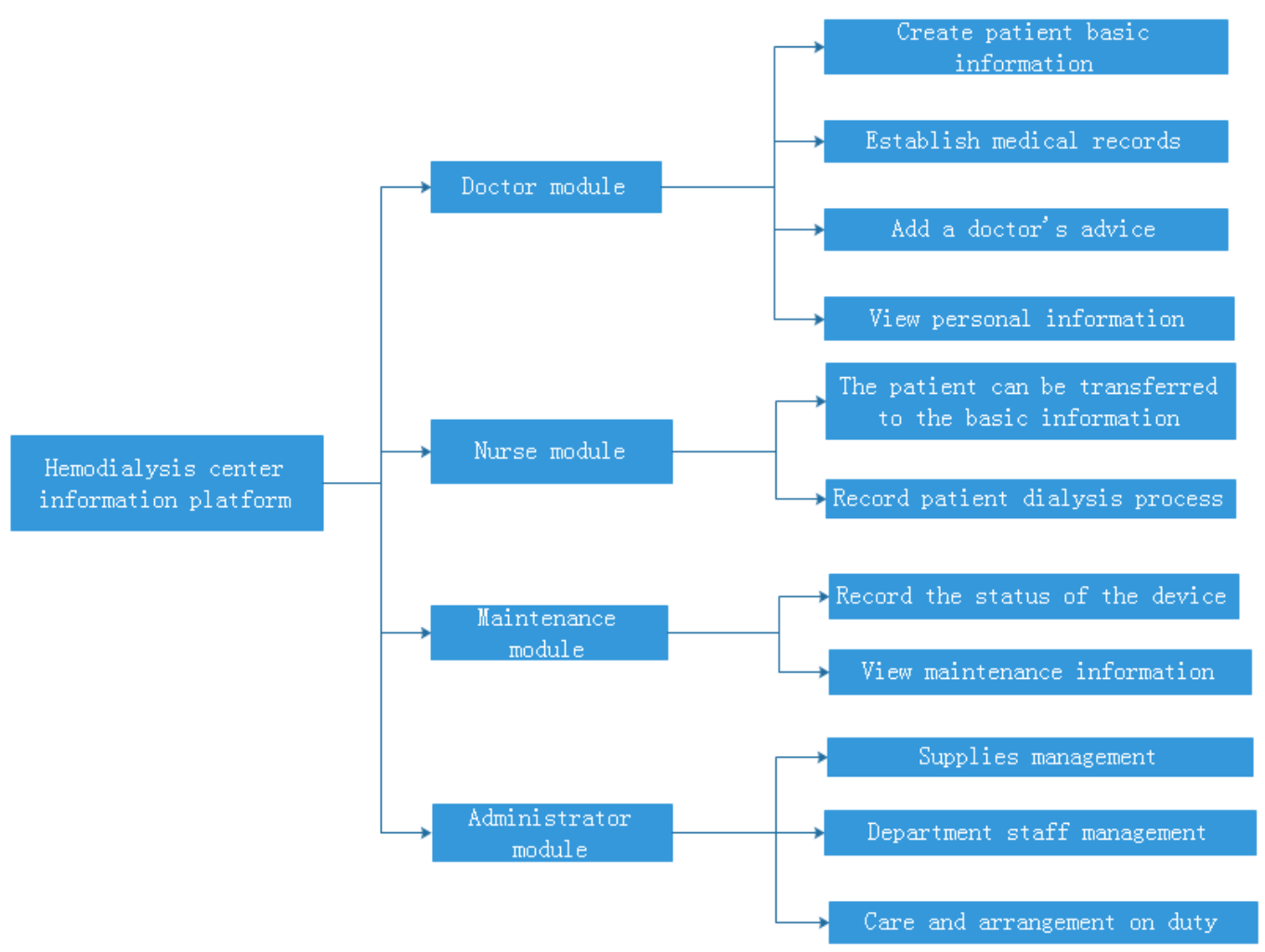

Figure 2: System module diagram

\subsubsection{Doctor function module}

Can create the basic information of patients, the establishment of medical records, add a doctor's advice, including long-term doctor's advice and temporary doctor's advice, each dialysis to develop dialysis program to see personal duty.

\subsection{2. $\quad$ Nurses function module}

Can be transferred to the patient basic information, long-term doctor's advice and temporary doctor's advice, dialysis treatment program and other information; can be the first patient before dialysis visit records; can record the patient during dialysis blood pressure, pulse, arteriovenous pressure and other parameters and dialyzer use Happening.

\subsubsection{Maintenance function module}


Maintenance of the main responsible for the hemodialysis center of the relevant medical equipment management, through the system can record the status of equipment and maintenance, you can record the device name, equipment number, brand, specifications, equipment serial number, purchase time, purchase price, registration Card number, whether free, maintenance and other information.

\subsubsection{Administrator function module}

Administrator module features include: hemodialysis center supplies management, department staff management and arrangements on duty. Supplies management mainly includes various types of dialyzers, dialysis fluid, blood vessels, puncture and other medical supplies within the needle, and to achieve a simple out of library management. The management of the staff of the department, the main information, including the job number, name, age, gender, occupation category, education, mobile phone number, title, graduation school, etc., and can manage the staff and can carry out the row Class, arranged on duty is mainly the administrator according to the actual situation and the frequency of staff on duty arrangements.

\section{CONCLUSIONS}

With the development of computer technology, hospital information management system has entered into all aspects of the hospital, medical equipment for the information management software system is also increasing, but for hemodialysis management information platform is relatively small. This article is based on the actual work of our hospital hemodialysis center based on the development, is fully aware of the needs of heal th care and engineering and technical personnel to achieve. The system for the nature of the work of various types of staff divided into different functional modules, through the system doctors can achieve the creation and management of patient information, you can handle the doctor's advice and the development of dialysis program nurses can call patient information and doctor's advice, the implementation of medical advice and Monitoring and control of the patient during the dialysis treatment of patient signs data and dialysis machine status data; engineering and technical personnel can effectively manage the hemodialysis center equipment; the system at the same time using serial communication way to hemodialysis equipment, a variety of parameters focused on the software The central station and stored records to the nursing module; department management staff can be hemodialysis center supplies and department staff to manage.

\section{ACKNOWLEDGEMENT}

Guangxi young teachers in the basic ability to enhance the project (KY2016LX229).

\section{REFERENCES}

[1] Jun, Z., Jingwei, F., Xiangfeng, Y. 2012. Hemodialysis center information management practice and discussion [J]. Chinese Medical Equipment, 27 (2), 97-98, 137.

[2] Wei, P., Yubin, Z. 2011. Hemodialysis center information management system key technology research [J]. China Digital Medicine, ,6 (7), 34-36.

[3] Sheng, Z. 2008. Network environment under the hemodialysis center information management system design and implementation [J]. Hospital Digital, 23 (2), 23-25.

[4] Hongjun, Z., Hui, X., Youping, X. 2007. Analysis of hemodialysis management system requirements. Medical information, 4 (20), 538-540.

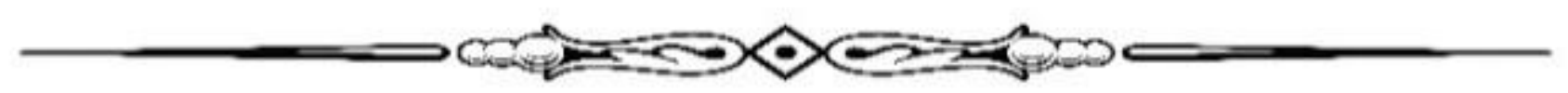

